\title{
Room Temperature Direct and Heterodyne Detection of 0.28-0.69-THz Waves Based on GaN 2-DEG Unipolar Nanochannels
}

\author{
Carlos Daher, Jérémie Torres, Ignacio Iñiguez-de-la-Torre, Philippe Nouvel, Luca Varani, Paul Sangaré, \\ Guillaume Ducournau, Christophe Gaquière, Javier Mateos, and Tomás González
}

\begin{abstract}
An experimental demonstration of GaN-based asymmetric nanodiodes as direct and heterodyne detectors up to $0.69 \mathrm{THz}$ has been performed at room temperature. Responsivities of 2 and $0.3 \mathrm{~V} / \mathrm{W}$ in a free-space configuration were obtained at 0.30 and $0.69 \mathrm{THz}$, respectively. An intermediate frequency (IF) signal has been measured up to 40 and $13 \mathrm{GHz}$ in the same frequency ranges. The characterization of the nanodiodes as mixers did not show any deviation from linearity between the RF input power and the IF output. Monte Carlo simulations, used to estimate nanodevice intrinsic conversion losses of $27 \mathrm{~dB}$ at $0.69 \mathrm{THz}$, have confirmed these results.
\end{abstract}

Index Terms-Frequency conversion, microwave circuits, mixers, sensor systems and applications detectors semiconductor detectors.

\section{INTRODUCTION}

D IRECT detection, one of the possible homodyne methods, is based on detecting a frequency-modulated continuous-wave radiation by a nonlinear device, which is able to rectify an alternating current, and generating - as a resulta dc voltage. In practice, the detection is often realized using a square-wave reference the so-called switching-mode detection. This method is widely used in optical applications [1] and radio communications [2]. Synchronous detection enables

Manuscript received May 26, 2015; revised October 23, 2015; accepted November 22, 2015. Date of current version December 24, 2015. This work was supported in part by the Seventh Framework Programme under Grant 243845, in part by the Dirección General de Investigación Científica y Técnica within the Ministerio de Economía y Competitividad under Project TEC2013-41640-R, and in part by the Consejería de Educación de la Junta de Castilla y León under Project SA052U13. The work of P. Sangaré, G. Ducournau, and C. Gaquière was supported in part by the electrical RF/MEMS characterization platform and in part by the ROOTHz European Project. The review of this paper was arranged by Editor S. Bandyopadhyay. (Corresponding author: Jérémie Torres.)

C. Daher, J. Torres, P. Nouvel, and L. Varani are with the Centre National de la Recherche Scientifique, Institut d'Electronique et des Systèmes, University of Montpellier, Montpellier 34090, France (e-mail: daher@ies.univ-montp2.fr; jeremi.torres@ies.univ-montp2.fr; nouvel@ies.univ-montp2.fr; luca.varani@ univ-montp2.fr).

I. Iñiguez-de-la-Torre, J. Mateos, and T. González are with the Department of Applied Physics, University of Salamanca, Salamanca 37008, Spain (e-mail: indy@usal.es; javierm@usal.es; tomasg@usal.es).

P. Sangaré, G. Ducournau, and C. Gaquière are with the Institut d'Electronique, de Microlectronique et de Nanotechnologie, University of Lille 1, Lille F-59100, France (e-mail: pa.sangare@ed.univ-lille1.fr; guillaume.ducournau@iemn.univ-lille1.fr; christophe.gaquiere@iemn. univ-lille1.fr).

Color versions of one or more of the figures in this paper are available online at http://ieeexplore.ieee.org.

Digital Object Identifier 10.1109/TED.2015.2503987 processing of very weak signals, even of amplitude lower than noise. It requires a reference signal with known frequency and phase, but it cannot give information about the frequency of the processed signal. An alternative method to detect a signal is the heterodyne detection by a nonlinear mixer device with a radiation of a referenced frequency. This technique, commonly used in telecommunications and astronomy for detecting and analyzing weak signals, also represents a very convenient way to detect terahertz $(\mathrm{THz})$ radiations. Indeed, heterodyne detection allows the use of conventional microwaves systems (such as spectrum analyzers) and simplifies the signal postprocessing by down-converting the high-frequency (HF) radiation $f_{\mathrm{RF}}$ (usually named $\mathrm{RF}$ signal) into a signal with an intermediate frequency (IF) $f_{\mathrm{IF}}$ falling into the microwave range by the help of an already known radiation [local oscillator (LO)] $f_{\mathrm{LO}}$ using the relation: $f_{\mathrm{IF}}=\left\|f_{\mathrm{RF}}-f_{\mathrm{LO}}\right\|$ [3]. The state-of-the-art electronic components for direct or heterodyne detections of an HF radiation are achieved by Schottky diodes [4]-[7]. Since these components are based on vertical transport, they require advanced materials, challenging fabrication processes, and critical technology aspects (air bridge to connect the anode introduces more complexity to the fabrication procedure and related parasitic elements) to operate at $\mathrm{THz}$ frequencies. More than the mainstream technologies, such as Schottky diodes, the emerging plasma-based detectors [8] also represent a promising alternative for future developments of THz mixers. Based on a different concept, the asymmetric nanodiodes have been proposed to obtain planar devices with nonlinear, diodelike, current-voltage $(I-V)$ characteristic [9]. Moreover, the use of high-mobility semiconductors, such as InGaAs [10] or InAs [11], can improve the cutoff frequency of these nanochannels, thus reaching the $\mathrm{THz}$ range at room temperature. However, it should also be possible to achieve $\mathrm{THz}$ operations with more robust materials, such as GaN. Even if the use of unipolar $\mathrm{GaN}$ nanochannels as $\mathrm{THz}$ detectors [12] or mixers [13] is fairly new, it presents a lot of room for improvements to make them competitive with a Schottky technology. In a previous paper, through a systematic numerical study based on Monte Carlo (MC) simulations, we have presented the performances of such $\mathrm{GaN}$ nanodiodes as direct and heterodyne detectors in the $0.30-\mathrm{THz}$ frequency range [13]. The aim of this paper is to present, over a wider 


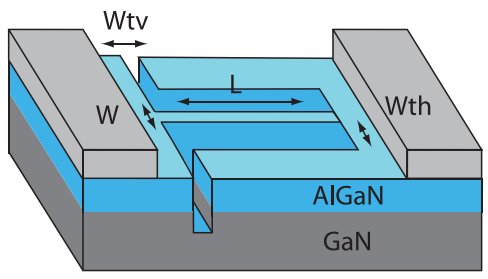

Fig. 1. 2-DEG unipolar nanochannels with a broken symmetry geometry. $W$ and $L$ stand for the channel width and length, and $W_{\mathrm{th}}$ and $W_{\mathrm{tv}}$ are the widths of the horizontal and vertical trenches, respectively.

frequency range, a systematic experimental study of the performances of GaN nanochannels as direct and heterodyne detectors with particular attention to their dependence on geometry, bias conditions, and frequency of operation. In particular, we show for the first time that GaN nanochannels can be used to detect a radiation at such HFs as $0.69 \mathrm{THz}$. This paper is organized as follows. Section II introduces the geometries of the different $\mathrm{GaN}$ nonlinear nanochannels, the experimental setups, and the MC model. Their performance at frequencies not reported so far $(0.64-0.69 \mathrm{THz})$ is presented in Section III-A and extended to a heterodyne configuration in Section III-B. Finally, the conclusions are given in Section IV.

\section{EXPERIMENTAL DETAILS}

\section{A. Samples Description}

The samples used are based on two dimensional Electron Gas unipolar nanochannels with a broken symmetry created by etching in the $\mathrm{AlGaN} / \mathrm{GaN}$ heterojunction two symmetrical L-shaped trenches (see Fig. 1 and [12] for technological details). Three geometrical configurations with $L / W=$ $500 / 500 \mathrm{~nm}, L / W=500 / 750 \mathrm{~nm}$, and $L / W=1000 / 750 \mathrm{~nm}$ have been tested, where $L$ and $W$ stand for the channels length and width, respectively. Vertical and horizontal trenches are $W_{\mathrm{tv}}=100 \mathrm{~nm}$ and $W_{\mathrm{th}}=50 \mathrm{~nm}$, respectively. To reduce the impedance of our nanodevices, 32 nanochannels were disposed in parallel without the need of any interconnection. In this way, the extrinsic parasitic elements can be limited, and this is an important feature at these frequencies. We emphasize that the simplicity of the technological process used for the fabrication of these nanodiodes is remarkable, since it only involves the etching of insulating recess lines on a semiconductor surface. Another key aspect of these nanodevices is their planar geometry, which provides the important advantages for the freespace radiation coupling over the traditional diodes (a Schottky barrier) used in current $\mathrm{THz}$ systems. In order to use these devices in a free-space configuration, planar bow-tie $\mathrm{THz}$ antennas were monolithically integrated with the devices. The bow-ties antennas have been designed to be very broadband, at least from 200 to $700 \mathrm{GHz}$ to be compatible with selfswitching devices. Each group of SSD devices was integrated at antenna feed-point, and dc line is feeding each bow-tie ends. The planar design of these antennas enables an SSD array configuration. The use of a large Si lens or an array of Si lenses could also enhance the performances, but for that a dedicated Si-lens array would be required and each signal $(\mathrm{LO}+\mathrm{RF})$ would have to be spatially overlapped to feed the mixer.

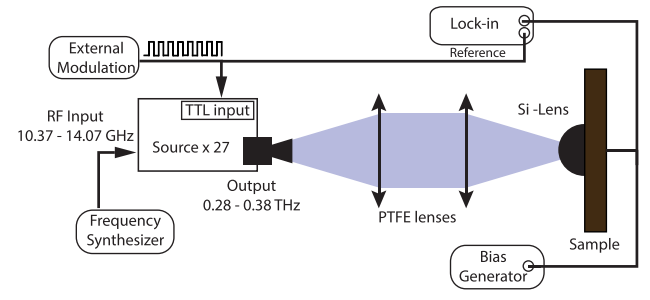

Fig. 2. Direct detection setup using the $0.3-\mathrm{THz}$ range source.

\section{B. Experimental Setups}

1) Direct Detection: To highlight the ability of these devices to detect a $\mathrm{THz}$ radiation, we have used two electronic sources from Virginia Diodes Inc. (VDI) to cover a large frequency range in experiments. One is a VDI-AMC-S222 tunable from 0.28 to $0.38 \mathrm{THz}$, and the other is a VDI-AMC-381 tunable from 0.64 to $0.69 \mathrm{THz}$ (Fig. 2). As this kind of electronic sources is composed of a multiplication chain, the measurement frequency points have been verified to be spurious free (e.g., monochromatic signal). At each frequency point, the sources output power was first calibrated using a VDI Erickson PM4 calorimeter matched with a WR10 waveguide load as input. Since we are measuring signals above this waveguide band $(59 \mathrm{GHz})$, adaptation is made by inserting a linear waveguide taper between the source output and the meter input. For the $0.30-\mathrm{THz}$ range source, we used a WR2.8 waveguide taper and measured a power that varies between 0.6 and $1 \mathrm{~mW}$ according to the frequency. For the $0.65-\mathrm{THz}$ range source, we used a WR1.2 waveguide taper and measured a power that varies between 1 and $1.3 \mathrm{~mW}$ according to the frequency. The RF input of the source is connected using a coaxial cable with subminiature version A (SMA) connectors to a frequency synthesizer Anritsu MG 3692B type, which delivers the microwave signal. This latter will be multiplied by the source. The $\mathrm{THz}$ signal is modulated at $1-\mathrm{kHz}$ frequency by an external modulation using an Agilent 33210A function generator connected to the Transistor-Transistor logic input of the source using a coaxial cable with Bayonet Neill-Concelman (BNC) connectors. First, the beam is collimated using a planoconvex polytetrafluoroethylene (PTFE) optical lens of 5-cm diameter and $10-\mathrm{cm}$ focal, and then, it is focused on the sample by another PTFE lens with the same characteristics. Finally, a silicon hemispherical lens of a 6-mm diameter is placed at the rear side of our sample to improve the focalization on each device, to reduce the free-space coupling losses, and to limit interferences inside the Si-bulk layer. The sample is mounted on an FR4-holder, especially designed for $50-\Omega$ impedance matching in the $20-\mathrm{GHz}$ frequency range. The antennas of the devices are connected through gold microbon dings to printed HF lines. The induced photovoltage is measured using a Signal Recovery 7265 digital signal processing lock-in amplifier, and the dc signal is averaged for each measurement to limit the noise effects. The biasing of the sample is done by a current supplier Keithley type connected to the device by a coaxial cable with BNC connectors. 


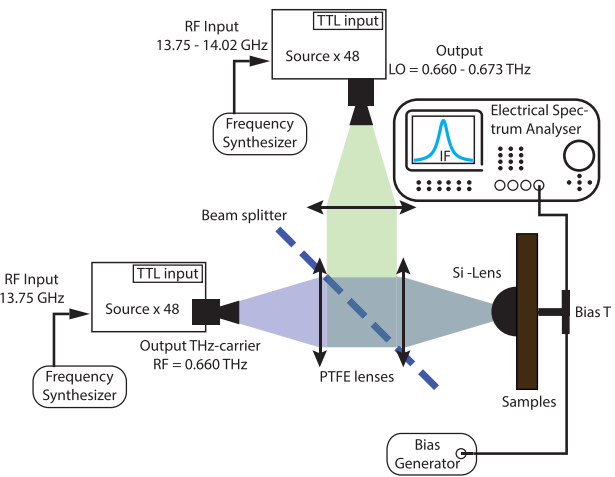

Fig. 3. Heterodyne detection setup using two $0.65-\mathrm{THz}$ range sources.

2) Heterodyne Detection: After ascertaining, using direct configuration, that a device is able to detect a $\mathrm{THz}$ radiation, we have studied his ability to mix two $\mathrm{THz}$ radiations by modifying the direct detection setup (Fig. 3). To perform heterodyne detection in the $0.30-\mathrm{THz}$ range, we used in addition to the $\mathrm{THz}$ source VDI-AMC-S222 type another $\mathrm{THz}$ source VDI-AMC-S163 type. This latter has a multiplication factor of 18 and covers the frequency range between 0.22 and $0.32 \mathrm{THz}$. Its measurement frequency points have been verified to be spurious free and at each frequency point, its output power was calibrated using the VDI Erickson PM4 calorimeter and a WR3.4 linear waveguide taper. We measured a power that varies between 0.6 and $1.6 \mathrm{~mW}$ according to the frequency. This source acts as RF frequency provider and is fixed at $0.29 \mathrm{THz}$, and the second source acts as the LO and its frequency varies between 0.29 and $0.33 \mathrm{THz}$. To perform heterodyne detection in the $0.65-\mathrm{THz}$ range, we used two identical VDI-AMC-381 THz sources, as shown in Fig. 3. The former one is fixed at $0.66 \mathrm{THz}$ and used as the RF signal, and the second one acts as the LO and its frequency varies between 0.66 and $0.69 \mathrm{THz}$. These two radiations are collimated and focused using PTFE lenses, whereas a polyethylene terephthalate beam splitter helps to spatially superimpose the two radiations. The Si-lens is still used. A bias-tee, with a bandpass of $50 \mathrm{MHz}-26 \mathrm{GHz}$, is connected to the SMA connectors of the holder in order to provide the dc-bias to the devices (fed by a dc-current source) and to measure the IF, with the help of a Rohde \& Schwartz FSU electrical spectrum analyzer. In those experiments, the IF can be measured up to $40 \mathrm{GHz}$ due to the limitation of our spectrum analyzer. In all cases, the experiments were performed in free-space and room temperature configurations. In order to understand the physical mechanisms and to optimize the performances of the devices for broadband direct detection (in terms of responsivity) and mixing (in terms of output power), a semiclassical MC simulation self-consistently coupled with a Poisson solver is used as previously explained in [13].

\section{RESULTS AND DISCUSSION}

\section{A. Direct Detection}

Since for direct detectors, the electrical rectification of an incident electromagnetic radiation is realized through the nonlinearities of the $I-V$ characteristic, we have plotted in Fig. 4, the $I-V$ characteristic of 32 nanochannels with

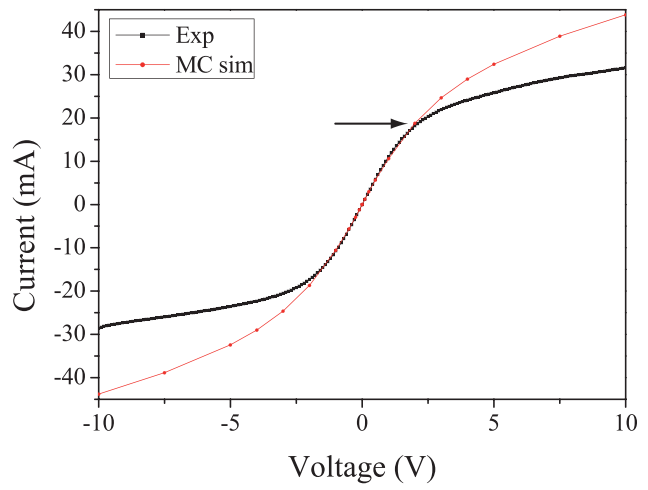

Fig. 4. Comparison between experimental $I-V$ characteristic of 32 nanochannels with $L / W=500 / 500 \mathrm{~nm}$ (black line and squares) and MC simulation (red line and dots).

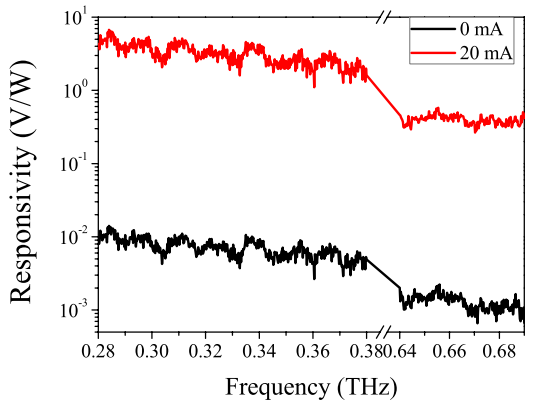

Fig. 5. Experimental responsivity in $V / W$ measured between 0.28 and $0.69 \mathrm{THz}$, by an array of 32 nanochannels with $L / W=500 / 500 \mathrm{~nm}$, with 0 - and $20-\mathrm{mA}$ bias currents.

$L / W=500 / 500 \mathrm{~nm}$. The black curve was experimentally obtained, whereas the red one results from MC simulations. The agreement between MC and measurements is very good in the low bias region showing as well the expected dependence with $W$ and $L$. However, some discrepancies appear at higher biases. We attribute the differences in the saturation regime to surface charge effects. These discrepancies at the dc level are responsible, latter in this paper, of some differences for the direct and heterodyne detections between MC and experiments.

In order to relate the effect of the bias current on the detector's performances, Fig. 5 shows the measured responsivity between 0.28 and $0.69 \mathrm{THz}$ of an array of 32 nanochannels with $L / W=500 / 500 \mathrm{~nm}$. The plotted responsivities are directly calculated as the ratio between the measured dc signal and the power of the source. Using a current of $20 \mathrm{~mA}$, we clearly observe a responsivity increase of three orders of magnitude, while its frequency dependence remains the same.

To better understand this behavior, we have studied in two frequency ranges $(0.29$ and $0.66 \mathrm{THz})$ the responsivity dependence with the current and compared it with the theoretical matched responsivity. The latter is equal to $1 / 2 R_{0} \gamma$, where $R_{0}$ is the zero-bias resistance and $\gamma$ is the curvature coefficient defined as $\gamma=\left(d^{2} I / d V^{2}\right) /(d I / d V)$ [14], [15].

Fig. 6 shows a very good agreement-for both frequency ranges - between the current dependence of the responsivity and that of the curvature coefficient. The application of 

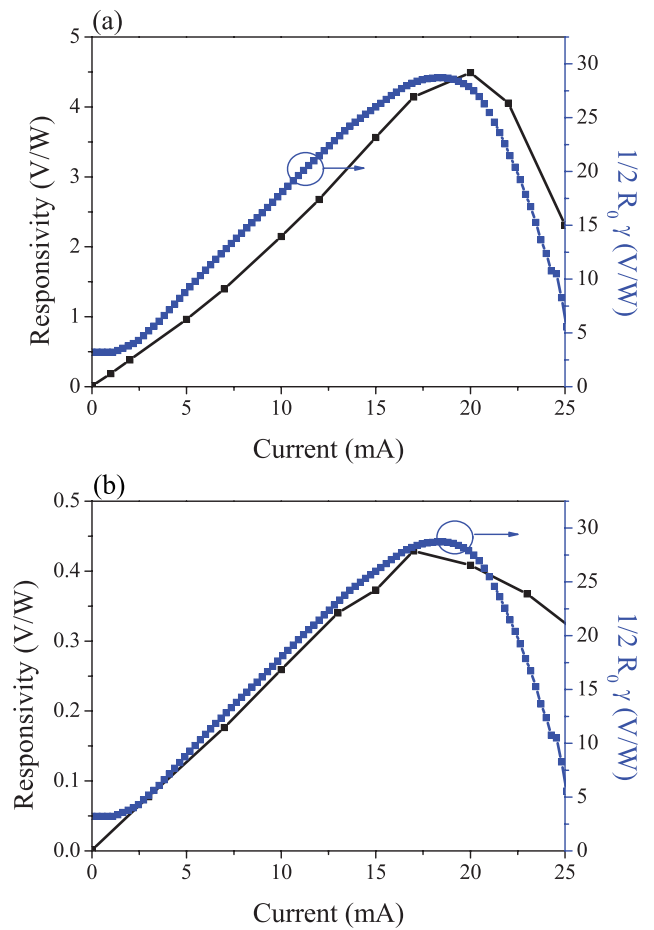

Fig. 6. Experimental responsivity in $V / W$ and $I-V$ curvature versus current at (a) 0.29 and (b) $0.66 \mathrm{THz}$ of an array of 32 nanochannels with $L / W=500 / 500 \mathrm{~nm}$.

an external current leads to higher values of the curvature coefficient and, therefore, to a higher voltage responsivity with an almost constant signal-to-noise ratio $S / N=12 \pm 1$ in the $0.30-\mathrm{THz}$ range and $S / N=2.9 \pm 0.3$ in the $0.65-\mathrm{THz}$ range. We notice that the maximum nonlinearity of the $I-V$ characteristic of our device is situated between 17 and $20 \mathrm{~mA}$ that is just in the knee of the $I-V$ curve of the nanochannel (indicated by an arrow in Fig. 4), as expected from the well-known link between the RF detection and the nonlinearity of the $I-V$ curve.

Fig. 7(a) shows the responsivities measured between 0.28 and $0.69 \mathrm{THz}$ in three arrays of 32 nanochannels each with different values of $L / W$ and with the same bias current of $10 \mathrm{~mA}$. Fig. 7 shows how reducing the width from 750 down to $500 \mathrm{~nm}$ significantly enhances the responsivity up to almost $2 \mathrm{~V} / \mathrm{W}$ at low frequency, as a result of more prominent surface effects that increase the nonlinearity of the $I-V$ characteristics. On the other hand, the length of the devices hardly affects the value of the responsivity. An important frequency roll-off is observed in all the cases ( $\sim 20 \mathrm{~dB} /$ decade), regardless of the channel geometry, but a strong cutoff is not clearly evidenced by the measurements. Measurements of the beam intensity at 0.30 and $0.65 \mathrm{THz}$ have been performed using a power-meter placed: 1) at the output of the source and 2) at the place where the sample is located. These measurements have allowed us to attribute this frequency dependence mainly to an increase of the optical losses at HF. This is also confirmed because of the MC results shown in Fig. 7(b). The frequency roll-off of these results, which do not consider the optical losses, is much lower (between 2 and $4 \mathrm{~dB} /$ decade), with a slightly more pronounced
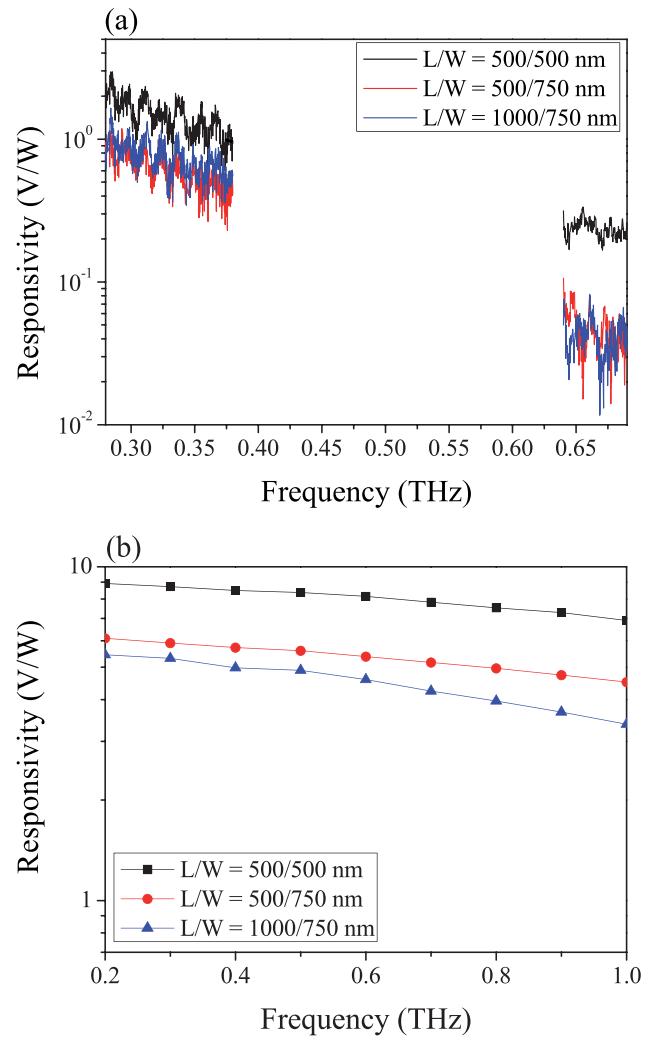

Fig. 7. (a) Experimental responsivity in $V / W$ measured between 0.28 and $0.69 \mathrm{THz}$, by three arrays of 32 nanochannels with different lengths and widths, with 10-mA bias current. (b) MC simulated intrinsic responsivities for the same devices.

roll-off in the case of $L=1000 \mathrm{~nm}$ (independently of the width of the channel). A good compromise between length and width is found for $W=500 \mathrm{~nm}$ and $L=500 \mathrm{~nm}$, providing an experimental responsivity above $0.2 \mathrm{~V} / \mathrm{W}$ up to almost $0.69 \mathrm{THz}$. At $0.30 \mathrm{THz}$, these experiments made in a free-space configuration provide a responsivity of $\sim 2 \mathrm{~V} / \mathrm{W}$, while the result of MC simulations in guided configuration (on-wafer) is $9 \mathrm{~V} / \mathrm{W}$. This comparison allows to estimate the optical losses induced by the coupling of the $\mathrm{THz}$ radiation with the device (THz focusing, PTFE and Si lenses, and wave propagation in free space) to be about $6.5 \mathrm{~dB}$ at low frequency. However, since we did not perform any data normalization related to the beam spot and the device sizes (which is very difficult to estimate and originates a lot of uncertainty), the real responsivities are probably higher than those reported. Moreover, our maximum detection frequency of $0.69 \mathrm{THz}$ may not represent the upper operational limit of our devices, since as shown by MC simulations, these devices could still be used at frequencies above $1 \mathrm{THz}$.

\section{B. Heterodyne Detection}

The IF power as a function of frequency is presented in Fig. 8 for the RF and LO signals falling into the $0.30-\mathrm{THz}$ range and $0.65-\mathrm{THz}$ range for an array of 32 nanochannels with $L / W=500 / 500 \mathrm{~nm}$ for different values of the current. In both cases, a strong attenuation around $13 \mathrm{GHz}$ is observed. This effect cannot be attributed to the cutoff frequency of 

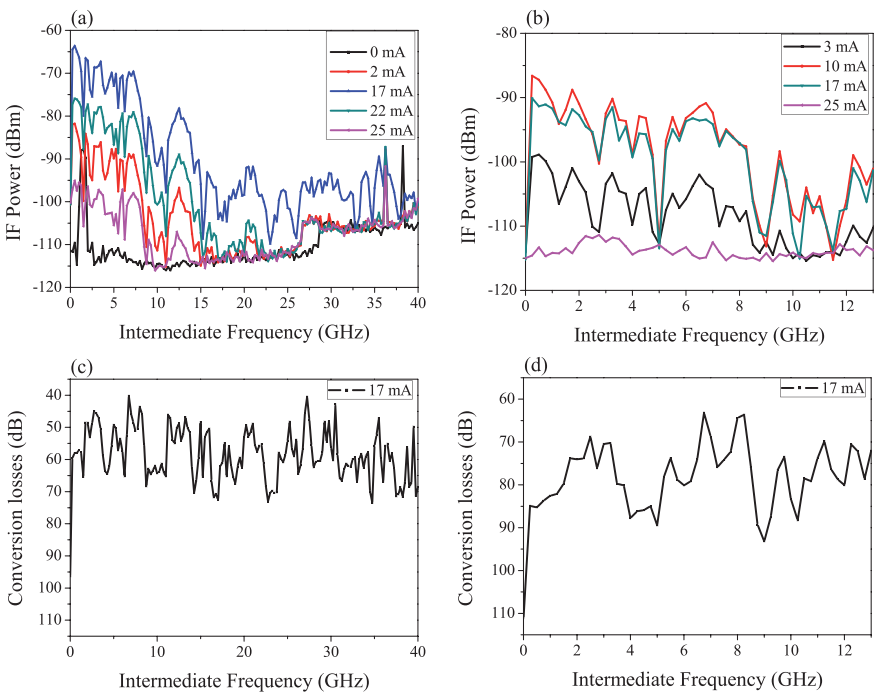

Fig. 8. Experimental IF power measured on a device with $L / W=500 / 500 \mathrm{~nm}$, for different currents, with RF and LO belonging to the frequency ranges (a) 0.30 and (b) $0.65 \mathrm{THz}$. (c) and (d) Corresponding CLs for $I=17 \mathrm{~mA}$.

our device. Indeed, the important figures of merit to be considered are the conversion losses (CLs) of our mixer. These have been determined as the ratio of the power at the IF frequency to the power at the RF frequency subtracting the losses induced by connecting elements and HF lines that were simulated using CST microwave studio and present a cutoff frequency around $13 \mathrm{GHz}$. Consequently, the CL can be plotted according to the IF [Fig. 8(c) and (d)] at 0.30- and 0.65-THz ranges, respectively, for the best bias conditions of $I=17 \mathrm{~mA}$. The CLs of the whole integrated device are $58 \pm 2 \mathrm{~dB}$ at $0.30-\mathrm{THz}$ range and increase by an additional $20 \mathrm{~dB}$ at higher frequencies to achieve $78 \pm 3 \mathrm{~dB}$ at $0.65-\mathrm{THz}$ range. Since CLs are mainly constant in a frequency range (e.g., 0.30 or $0.65 \mathrm{THz}$ ), one can conclude that the bandwidth of our mixer is at least of $40 \mathrm{GHz}$, i.e., the frequency limit of our electrical spectrum analyzer. These values do not represent the intrinsic CL of the devices, since, as for the responsivity measurements, the losses due to radiation coupling with the antenna (especially in the $0.65-\mathrm{THz}$ frequency range) and bondings wires are difficult to estimate experimentally. Using the same method, CL has been calculated for each current, and the results for an IF of $1 \mathrm{GHz}$ (while RF and LO belong to the $0.65-\mathrm{THz}$ range) are plotted in Fig. 9. These results are then compared with the intrinsic CL obtained by an MC simulation done on a single nanochannel with $L / W=500 / 500 \mathrm{~nm}$. Notice that the simulation has been performed for an IF of $50 \mathrm{GHz}$ instead of $1 \mathrm{GHz}$ to decrease the calculation time (and providing the same result, since it is MC IF power is constant below $100 \mathrm{GHz}$ [13]). Fig. 9 shows good agreement between experimental and MC simulation results until a bias current of $17 \mathrm{~mA}$. The disagreement after this biasing point is due to discrepancies between experimental and MC $I-V$ characteristics (Fig. 4). For current lower than $17 \mathrm{~mA}$, by subtracting the intrinsic 27-dB losses calculated by the MC simulation to the

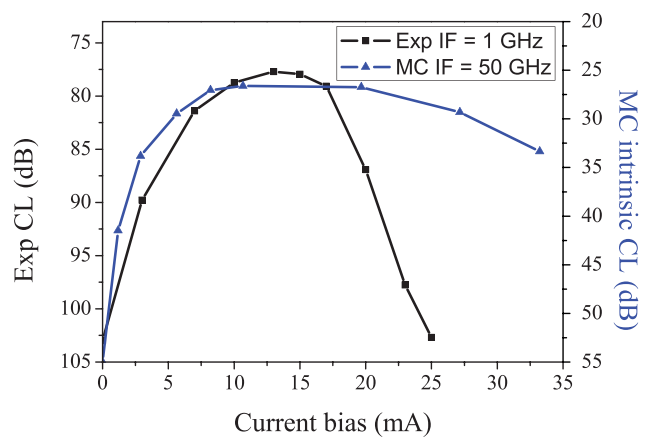

Fig. 9. Experimental power versus current of an IF $=1 \mathrm{GHz}$ and $\mathrm{MC}$ intrinsic CLs for an IF $=50-\mathrm{GHz}$ power versus bias current. In this case, a single nanochannel with $L / W=500 / 500 \mathrm{~nm}$ is simulated. The RF and LO signals belong to the $0.65-\mathrm{THz}$ range.

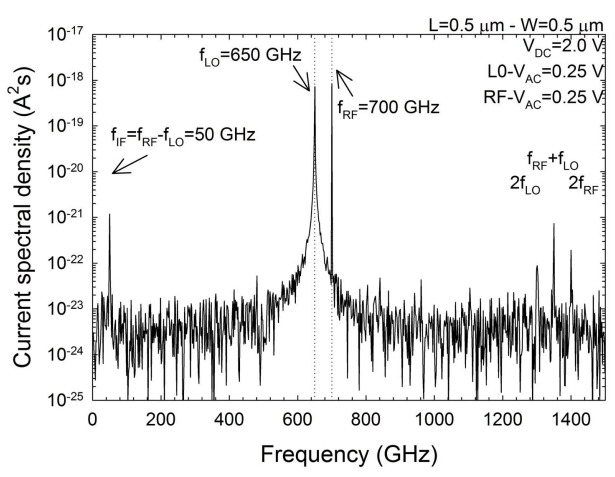

Fig. 10. MC simulated spectra of the current when the frequency of the LO and RF signals are 650 and $700 \mathrm{GHz}$, respectively, and the bias conditions are $\mathrm{VDC}=2 \mathrm{~V}$. In this case, a single nanochannel is simulated with $L / W=500 / 500 \mathrm{~nm}$.

experimental 78-dB losses, we can deduce that the experimental setup ( $\mathrm{THz}$ focusing, PTFE and Si lenses, and wave propagation in free space) and the device integration (bondings and contacts) introduce the additional losses of $\sim 51 \mathrm{~dB}$.

In Fig. 8(c) and (d), we have estimated that the IF bandwidth of our mixer is at least $40 \mathrm{GHz}$, which is the limitation provided by our instruments. To confirm this estimation, Fig. 10 highlights the current spectral density calculated for a single nanochannel with $L / W=500 / 500 \mathrm{~nm}$. First, Fig. 10 shows an IF bandwidth up to $50 \mathrm{GHz}$, since the LO frequency was chosen at $0.65 \mathrm{THz}$ and the RF was chosen at $0.70 \mathrm{THz}$. Second, the simulated device demonstrates no intrinsic frequency limitation up to $1.35 \mathrm{THz}$, since a peak corresponding to the upconversion case and with the same order of magnitude as the IF peak is obtained at the frequency $f_{\mathrm{UP}}=f_{\mathrm{RF}}+f_{\mathrm{LO}}=1.35 \mathrm{THz}$.

Finally, another figure of merit of our mixer is studied: the conversion compression, e.g., the maximum RF input power for which the mixer will provide linear operation in terms of constant CL. Fig. 11(a) shows the measurements of the IF output power dependence on the RF input power, whereas Fig. 11(b) presents the corresponding MC simulation results. From experiments, the RF power-measured at the devices top and after all optical elements-varies from -4 to $-2.13 \mathrm{dBm}$. Outside these limits, the power of the RF source is 

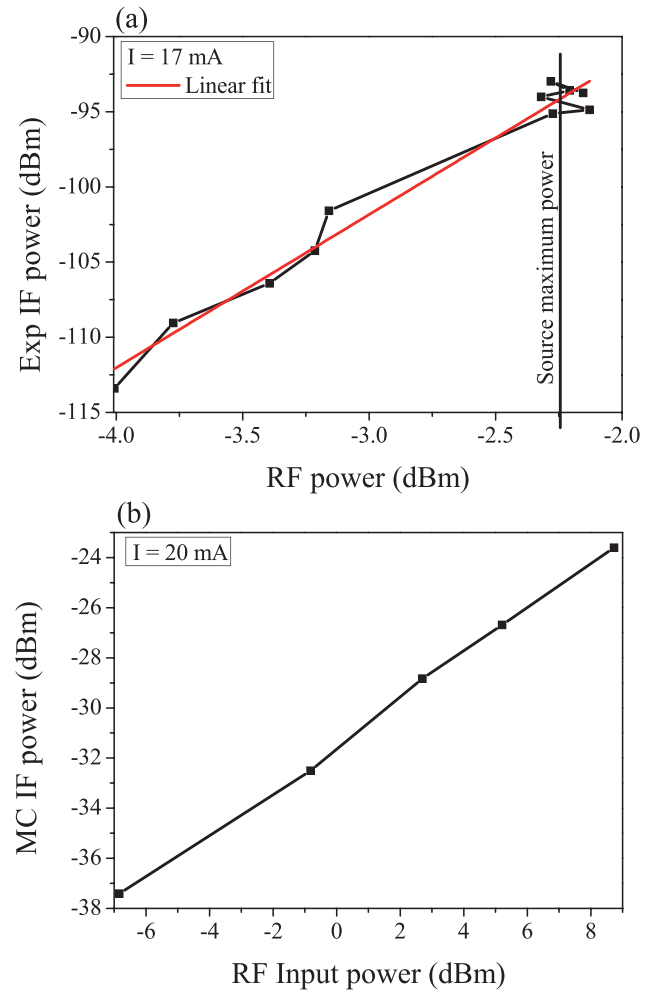

Fig. 11. (a) $\mathrm{RF}=0.660 \mathrm{THz}$ and $\mathrm{LO}=0.661 \mathrm{THz}$, experimental IF power versus RF input power measured with an array of 32 nanochannels with $L / W=500 / 500 \mathrm{~nm}$ and with a current bias of $17 \mathrm{~mA}$. Straight line: linear fit of the experimental measurements. (b) $\mathrm{RF}=0.70 \mathrm{THz}$ and $\mathrm{LO}=0.65 \mathrm{THz}$, MC simulation of the IF power versus RF input power done on the same device with a current bias of $20 \mathrm{~mA}$.

either OFF or reaches its maximum value. In both experimental and MC cases, a linear dependence is observed. From the experimental point of view, no saturation behavior has been observed up to the maximum available power of $-2.13 \mathrm{dBm}$. However, even MC simulations performed for much higher RF power (up to $9 \mathrm{dBm}$ ) did not show any saturation of the IF power. This robust linearity of the heterodyne detector response represents a clear advantage compared with the other $\mathrm{THz}$ mixers. The important point is that these GaN-based detectors can handle very high input RF power, so that their practical application as the power detectors of ultrahigh-power submillimeter-wave sources seems feasible.

\section{CONCLUSION}

In summary, we have investigated the potentialities of $\mathrm{AlGaN}-/ \mathrm{GaN}$-based nonlinear nanochannels for $\mathrm{THz}$ direct as well as for $\mathrm{THz}$ heterodyne detection. Their room temperature good responsivity as direct $\mathrm{THz}$ and homodyne detectors up to $0.69 \mathrm{THz}$ has been demonstrated for the first time. By comparison with MC simulations, we have found a good compromise between length and width of the nanochannels in order to optimize their responsivity as well as their frequency roll-off at higher frequencies. The qualitative good agreement between experiments and MC simulations will provide the guidelines for improving the SSD performances at $\mathrm{THz}$ frequencies.
The characterization of the nanodevices as $\mathrm{THz}$ mixers has permitted to highlight two main figures of merit: 1) CLs of $33 \mathrm{~dB}$ at $0.65 \mathrm{THz}$ and 2) a strong linearity of the IF output up to $8 \mathrm{dBm}$ of RF input power. As a consequence, since GaN-based nanodevices could handle very high input RF power without deviation from linear regime, their practical implementation as the mixers of high-power submillimeterwave sources seems feasible. Moreover, recent studies of similar GaN-based nanodiodes [16] have demonstrated their possibilities as Gunn oscillators that could be employed as the active elements of $\mathrm{THz}$ emitters. As a consequence, the good performances of $\mathrm{GaN}$ nanochannels demonstrated in this paper as the detectors of submillimeter-wave signals as well as their planar geometry allowing not only the easy integration of antennas for a better free-space coupling but also a flexibility in the design for an optimum thermal dissipation and reduction of parasitic effects open the possibility to develop a fully integrated emitter/detector submillimeterwave system working at room temperature. Besides, this SSD technology is compatible with a power amplifier association on the same dice (adjustment of the conversion gain). It means for heterodyne detection, to process on the same epilayer (heterostructure $\mathrm{AlGaN} / \mathrm{GaN}$ on a Si substrate) an IF amplifier and an SSD heterodyne mixer. This solution is very promising seeing the low cost solution and the mounting simplification.

\section{REFERENCES}

[1] A. Rakshit and A. Chatterjee, "A microcontroller-based compensated optical proximity detector employing the switching-mode synchronous detection technique," Meas. Sci. Technol., vol. 23, no. 3, p. 035102, 2012.

[2] J. Kumar and R. Mehra, "Costas loop implementation for synchronous detection of carrier in radio receiver," in Proc. Int. Conf. Adv. Comput., Commun. Netw., 2011, pp. 975-980.

[3] M. C. Teich, "The physics of heterodyne detection in the far-infrared: Transition from electric-field to photon-absorption detection in a simple system," Heterodyne Syst. Technol., vol. 100, pp. 1-10, Mar. 1980.

[4] H.-W. Hübers, "Terahertz heterodyne receivers," IEEE J. Sel. Topics Quantum Electron., vol. 14, no. 12, pp. 378-391, Mar./Apr. 2008.

[5] H. Kazemi et al., "Ultra sensitive ErAs/InAlGaAs direct detectors for millimeter wave and THz imaging applications," in Proc. IEEE/MTT-S Int. Microw. Symp., Jun. 2007, pp. 1367-1370.

[6] O. Cojocari et al., "A new structural approach for uniform submicrometer anode metallization of planar THz Schottky components," Semicond. Sci. Technol., vol. 19, no. 3, pp. 537-542, 2004.

[7] A. Semenov, O. Cojocari, H.-W. Hübers, F. Song, A. Klushin, and A.-S. Müller, "Application of zero-bias quasi-optical Schottky-diode detectors for monitoring short-pulse and weak terahertz radiation," IEEE Electron Device Lett., vol. 31, no. 7, pp. 674-676, Jul. 2010.

[8] D. Glaab, S. Boppel, A. Lisauskas, U. Pfeiffer, E. Öjefors, and H. G. Roskos, "Terahertz heterodyne detection with silicon fieldeffect transistors," Appl. Phys. Lett., vol. 96, no. 4, p. 042106, 2010. [Online]. Available: http://scitation.aip.org/content/aip/journal/apl/96/4/ $10.1063 / 1.3292016$

[9] A. M. Song, M. Missous, P. Omling, A. R. Peaker, L. Samuelson, and W. Seifert, "Unidirectional electron flow in a nanometer-scale semiconductor channel: A self-switching device," Appl. Phys. Lett., vol. 83, no. 9, pp. 1881-1883, 2003.

[10] I. Iñiguez-de-la Torre, H. Rodilla, J. Mateos, D. Pardo, A. M. Song, and T. González, "Terahertz tunable detection in self-switching diodes based on high mobility semiconductors: InGaAs, InAs and InSb," J. Phys., Conf. Ser., vol. 193, no. 1, p. 012082, 2009.

[11] A. Westlund et al., "Optimization and small-signal modeling of zero-bias InAs self-switching diode detectors," Solid-State Electron., vol. 104, pp. 79-85, Feb. 2015. [Online]. Available: http://www.sciencedirect.com/science/article/pii/S0038110114002822 
[12] P. Sangaré et al., "Experimental demonstration of direct terahertz detection at room-temperature in $\mathrm{AlGaN} / \mathrm{GaN}$ asymmetric nanochannels," J. Appl. Phys., vol. 113, no. 3, p. 034305, 2013. [Online]. Available: http://scitation.aip.org/content/aip/journal/jap/113/ 3/10.1063/1.4775406

[13] I. Íñiguez-de-la-Torre et al., "Operation of GaN planar nanodiodes as THz detectors and mixers," IEEE Trans. Terahertz Sci. Technol., vol. 4, no. 6, pp. 670-677, Nov. 2014.

[14] A. M. Cowley and H. O. Sorensen, "Quantitative comparison of solidstate microwave detectors," IEEE Trans. Microw. Theory Techn., vol. 14, no. 12 , pp. 588-602, Dec. 1966.

[15] Q. Zhou, K.-Y. Wong, W. Chen, and K. J. Chen, "Wide-dynamic-range zero-bias microwave detector using $\mathrm{AlGaN} / \mathrm{GaN}$ heterojunction fieldeffect diode," IEEE Microw. Wireless Compon. Lett., vol. 20, no. 15, pp. 277-279, May 2010.

[16] A. Íñiguez-de-la-Torre et al., "Searching for THz Gunn oscillations in GaN planar nanodiodes," J. Appl. Phys., vol. 111, no. 11, p. 113705 , 2012.

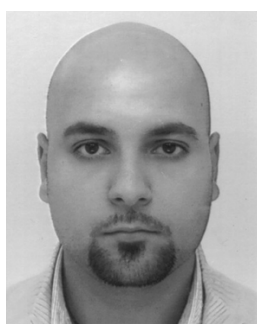

Carlos Daher was born in Thoum, Lebanon, in 1986. He is currently pursuing the Ph.D. degree with the Institute of Electronics and Systems, University of Montpellier, Montpellier, France.

$\mathrm{He}$ is involved in the development of new detectors and emitters of terahertz radiations based on selfswitching nanodevices.

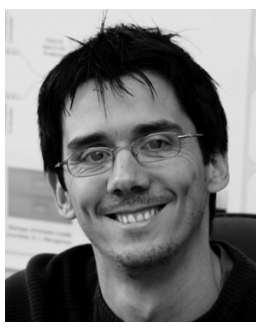

Jérémie Torres was born in 1977.

$\mathrm{He}$ is currently an Associate Professor of the Institute of Technology of Montpellier with the University of Montpellier, Montpellier. His research has been concerned with high-frequency electronics and transport in semiconductor materials and devices for generation, detection, and applications of terahertz radiations. He has authored and co-authored about 100 scientific articles in refereed journals and conference proceedings.

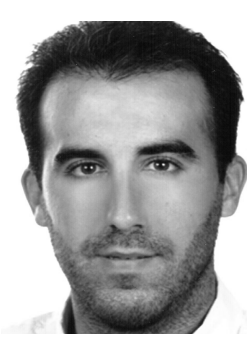

Ignacio Iñiguez-de-la-Torre was born in Valladolid, Spain, in 1981.

$\mathrm{He}$ is currently a Lecturer with the Electronics Group, Department of Applied Physics, University of Salamanca, Salamanca, Spain. His current research interests include development of novel device concepts for terahertz data processing, detection, and emission using both narrow and wide bandgap III-V semiconductors.

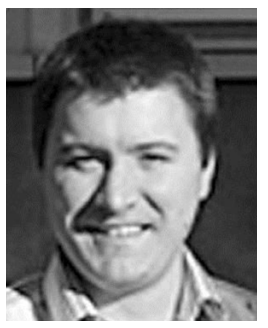

Philippe Nouvel was born in Nimes, France, in 1975.

$\mathrm{He}$ is currently a Project Engineer with the Centre National de la Recherche Scientifique, University of Montpellier, France. He is also with the Institute of Electronics of the South, Montpellier, France, where he is with the High Frequency Investigation Group. His current research interests include detection and generation of terahertz radiations by optoelectronic systems.

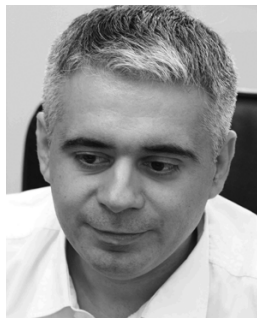

Luca Varani received the Ph.D. degree in physics from the University of Modena, Modena, Italy, and the $\mathrm{Ph} . \mathrm{D}$. degree in electronics from the University of Montpellier, Montpellier, France.

$\mathrm{He}$ is currently a Full Professor with the Institute of Electronics and Systems, University of Montpellier. His current research interests include theoretical and experimental transport phenomena in semiconductor materials and devices with a special attention to terahertz frequencies.

Paul Sangaré, photograph and biography not available at the time of publication.

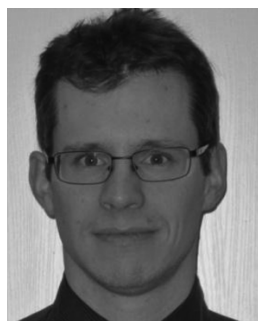

Guillaume Ducournau received the Ph.D. degree in fiber optic communication systems using DPSK modulation schemes from the University of Rouen, Mont-Saint-Aignan, France, in 2005.

He has been an Assistant Professor with the Institut d'Électronique de Microélectronique et de Nanotechnologie, University of Lille 1, Lille, France, and the Graduate School, Polytech'Lille, Villeneuve d'Ascq, since 2007.

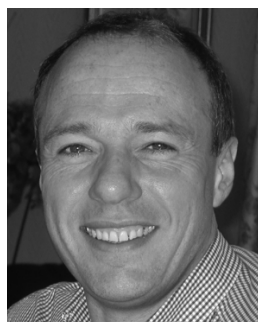

Christophe Gaquière received the Ph.D. degree in electronics from the University of Lille 1, Lille, France, in 1995.

$\mathrm{He}$ is currently a Full Professor with the University of Lille 1 , and carries out his research activity at the Institut d'Electronique de Microélectronique et de Nanotechnology. His current research interests include design, fabrication, characterization, and modeling of HEMTs and HBT devices.

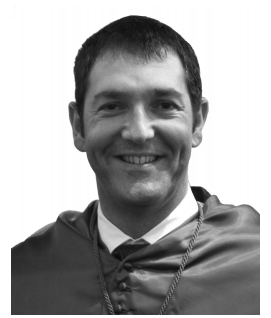

Javier Mateos was born in Salamanca, Spain, in 1970 .

He has been with the Department of Applied Physics, University of Salamanca, Salamanca, since 1993, where he became an Associate Professor in 2000. He has authored or co-authored over 100 refereed scientific journal papers and 150 conference contributions. His current research interests include the study of HEMTs based in both narrow and wide bandgap semiconductors.

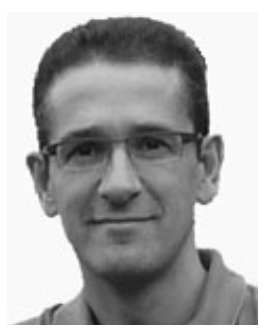

Tomás González was born in Salamanca, Spain, in 1967.

He has been with the Department of Applied Physics, University of Salamanca, Salamanca, since 1991, where he is currently a Full Professor of Electronics. His current research interests include highfrequency III-V transistors, microscopic modeling of electronic noise, and development of novel terahertz device concepts based on ballistic transport. 